\title{
Análisis comparativo de las variables físicoquímicas y bromatológicas de una composición alimenticia derivada de especies agrícolas nativas y un concentrado comercial para la cría de pollos de engorde
}

\section{Comparative analysis of the variables físicoquímicas and bromatológicas of a food composition derived from agricultural native species and a commer- cial concentrate for the baby of chickens of puts on weight}

\author{
Moisés Mosquera Blandón ${ }^{1}$, Juan Alberto Asprilla Abadía², Mirla Perea Murillo³
}

\section{Resumen}

A partir de estudios adelantados en las estaciones ambientales del Instituto de Investigaciones Ambientales del Pacifico (IIAP), se evaluaron dietas para la cría y ceba de pollos de engorde. Se diseñó, elaboró y validó una composición alimenticia obteniendo pesos promedios de 4.5 libras/animal/ ciclo. En este estudio se planteó un análisis comparativo de las variables fisicoquímicas y bromatológicas de esta composición alimenticia, basada en harina de maiz Chococito (25\%) y plátano (33\%), torta de soya (25\%), harina de hueso (10\%), carbonato de calcio (6\%), sal (1\%) y un concentrado comercial, como propuesta para generar un alimento alternativo efectivo y económico. Se aplicó la metodología descrita por Mosquera et al. (2008), para la elaboración de la composición alimenticia. La muestra enviada al laboratorio se tomó de acuerdo con el protocolo de la NTC-ISO/IEC 17025 (2005), se rotuló y se envió para los análisis respectivos. Los resultados muestran que la composición alimenticia contiene una alta concentración de cenizas o minerales totales (37.6\%), grasas totales $(24.8 \%)$, calorías totales $(329 \%)$, carbohidratos totales $(10.7 \%)$, humedad $(11.1 \%)$, proteina total (15.7\%), fibra (no determinada) y nitrógeno (2.5\%), valores que respecto al concentrado comercial cumple con las concentraciones requeridas para la alimentación y nutrición de explotaciones comerciales de pollos de engorde; se logró un ahorro de \$37.037 por ciclo productivo, generándose enormes posibilidades de utilizar esta dieta para la producción comercial de pollos de engorde con rentabilidades similares o superiores a las obtenidas con alimentos comerciales.

Palabras clave: Bromatología del maíz, Composición alimenticia, Maíz Chococito.

\begin{abstract}
On the basis studies advanced in the environmental stations of the Instituto de Investigaciones Ambientales del Pacifico (IIAP), on the design and evaluation of diets for feeding smaller species designed, developed and validated a food composition for rearing broiler weights obtaining averages $4.5 \mathrm{Lbs} /$ Pet/cycle. In this study a comparative analysis of physicochemical and qualitative variables of said food composition based on Chococito corn flour (25\%) and banana (33\%), soybean meal (25\%), bone meal (raised to $10 \%)$, calcium carbonate (6\%) and sal (1\%) and industrial concentrate, as proposed to generate an effective and economical alternative food. The
\end{abstract}

1 Ing. Agrónomo, Especialista en Gerencia de Recursos Naturales, Magister en Liderazgo y Gerencia Ambiental, Investigador Principal del Componente Productivo del Instituto de Investigaciones Ambientales del Pacífico (IIAP), Quibdó, Colombia. e-mail: mmosquera@iiap.org.co

2 Profesional en Química, Contratista Investigador del Componente Productivo del Instituto de Investigaciones Ambientales del Pacífico (IIAP), Quibdó, Colombia. e-mail: jasprill@gmail.com

3 Ing. Agroforestal, Investigador Asociado I del Instituto de Investigaciones Ambientales del Pacífico (IIAP), Quibdó, Colombia. e-mail: mperea@iiap.org.co

Recibido: 10 de diciembre de 2012 Aceptado: 19 de marzo de 2013 


\section{Bioetnia Volumen 10, 2013}

methodology described by B. Mosquera et al. (2.008) for the preparation of the food composition, the sample sent to the laboratory was made according to the protocol of the NTC-ISO /IEC 17025 (2005) was applied, was labeled and sent for the respective analysis. The results show that the food composition contains moisture (11.1\%), total protein (15.7\%), total fat (24.8\%), nitrogen (2.5\%) or total mineral ash (37.6\%), total carbohydrate (10.7\%), total calories (329\%), fiber (0\%) values compared to commercial concentrate meet the levels and concentrations required for food and nutrition commercial broiler farms, a savings of $\$ 37,037$ was reached pesos per production cycle this creates significant opportunities for using this diet for commercial production of broilers with close or similar results to those obtained with commercial foods.

Keywords: Alternative food composition, Chococito corn, Maize food science.

\section{Introducción}

La carne de pollo es altamente nutritiva, contiene proteína de alta calidad, vitaminas, potasio, calcio y fósforo, entre otros componentes y la cantidad de grasa es mínima, comparada con otras carnes como la vacuna y porcina. Debido a estos valores, es la carne preferida por las personas que cuidan su peso y aquellos que deben restringir su consumo en grasa. La carne de pollo forma parte de una dieta balanceada en la que existe una inmensa variedad de alimentos, necesarios para llevar una vida equilibrada y saludable (Friedman y Weil 2010).

A nivel mundial, la producción avícola, tiene como una de las mayores limitantes, la alimentación de las aves que incide fuertemente en el costo de producción, bien sean para pollos de engorde, ponedoras o reproductoras. El valor de la producción alimenticia tiene un porcentaje muy alto en los costos totales en el establecimiento de galpones. Para la reducción de estos costos se han tenido en cuenta y/o propuesto algunas alternativas a base de suplementos alimenticios a partir de cultivos nativos de las regiones donde se producen aves. El concepto de recurso alimenticio alternativo hace alusión a aquel no comúnmente empleado en la elaboración comercial de alimentos balanceados para animales y que puede sustituir o reemplazar parcialmente a las fuentes convencionales de nutrientes y de energía representadas en los granos de cereales, leguminosas y harinas de origen animal Berrio y Cardona (2001).

En Colombia y más en la región del Chocó Biogeográfico, se presenta una situación particular que hace aún más alto el costo de la producción de estas aves, debido al mal estado de las vías, la comercialización de pollos traídos desde otras ciudades y el alto costo de los alimentos para levante y ceba, lo que produce unos elevados precios de ventas para el consumidor final, de ahí la importancia de buscar alternativas más económicas para la producción de las aves.

Partiendo de la búsqueda de alternativas para garantizar los contenidos nutricionales y minimizar costos en la alimentación de pollos de engorde y a la vez, de obtener un producto final de buena calidad, el Instituto de Investigaciones Ambientales del Pacífico (IIAP), Quibdó, Colombia, realizó el análisis fisicoquímico y bromatológico de una composición alimenticia alternativa compuesta por harina de plátano, harina de maíz, harina de hueso, carbonato de calcio, torta de soya y sal, dieta que fue validada en años anteriores partiendo del éxito de las experiencias desarrolladas, sobre la evaluación de los efectos de algunos productos alimentarios tradicionales en la cría y levante de pollos en las estaciones ambientales del IIAP, los eonsejos comunitarios e instituciones agroambientales del Chocó Biogeográfico, con el fin de obtener un producto para comercializar a precios más bajos, con la misma o mejor calidad que los concentrados comerciales que se encuentran en el mercado local, regional, nacional e internacional.

\section{Metodología}

Área de estudio. El municipio de Quibdó, capital del departamento del Chocó, limita por el norte con el municipio de Medio Atrato, por el sur con los municipios de Río Quito y Lloró, por el oriente con el municipio de El Carmen de Atrato, por el nororiente con el departamento de Antioquia, por el occidente con el municipio de Alto Baudó, por el noroccidente con el municipio de Bojayá (Figura 1). La cabecera municipal de Quibdó se encuentra a $5^{\circ} 41^{\prime} 13^{`}$ de latitud norte y $76^{\circ} 39^{\prime} 40^{`}$ de longitud este, con respecto al meridiano Greenwich; la superficie territorial del municipio es de $3.075 \mathrm{~km}^{2}$, altura sobre el nivel de 43 msnm y temperatura promedio $28^{\circ} \mathrm{C}$ (Alcaldía Municipal de Quibdó 2011).

\section{Método}

Preparación de composición alimenticia. Se aplicó el método definido por Mosquera et al. (2008), según el siguiente procedimiento: adquisición de insumos, lavado, picado de plátano y maíz, pesado de las proporciones de plátano y maíz, precocción entre 10 y 15 minutos, secado entre 1 y 2 días dependiendo de la radiación solar, molido, mezcla con insumos minerales, sal y empacado. No se consideró la utilización de aminoácidos.

Toma y envío de la muestra. Se elaboró una dieta aproximadamente de $70 \mathrm{~kg}$; se tomaron muestras aleatorias y finalmente una muestra de un kilogramo de peso, de acuerdo con el protocolo de la NTC-ISO/IEC 17025 del 2005; luego la muestra se homogeneizó, se rotuló y se 
envió al laboratorio para los análisis fisicoquímicos, en procura de determinar y analizar los contenidos de proteína, grasa, humedad, cenizas, siguiendo la metodología propuesta por la Official Methods of Analysis (AOAC).

Análisis de laboratorio. Los análisis fisicoquímicos se realizaron en el Laboratorio de Salud Pública de la Universidad de Antioquia (Tabla 1).

Determinación de costos de preparación de la composición alimenticia. Se establecieron los costos totales de los insumos requerido para la preparación de la composición nutricional y se compararon con el valor o precios a que se comercializa el alimento concentrado en la ciudad de Quibdó.

\section{Resultados y discusión}

Los análisis de laboratorio mostraron los siguientes resultados para los parámetros físicos y bromatológicos, los cuales se discuten a continuación:

Parámetros físicos. En la Tabla 2 se consigna los resultados obtenidos en laboratorio sobre las condiciones físicas de la composición alimenticia en estudio.

Estos resultados muestran que no se encontraron diferencias con respecto al concentrado comercial; los aspectos físicos de la composición alimenticia como son la humedad, color y olor se encuentran dentro de los parámetros normales requeridos para este tipo de alimentos que son utilizados para la nutrición animal, lo cual genera ventajas comparativas con respecto al concentrado comercial porque no se requieren insumos adicionales para darle esta importante característica, básica para facilitar a las aves la ingesta del alimento y garantizar la comercialización del producto.

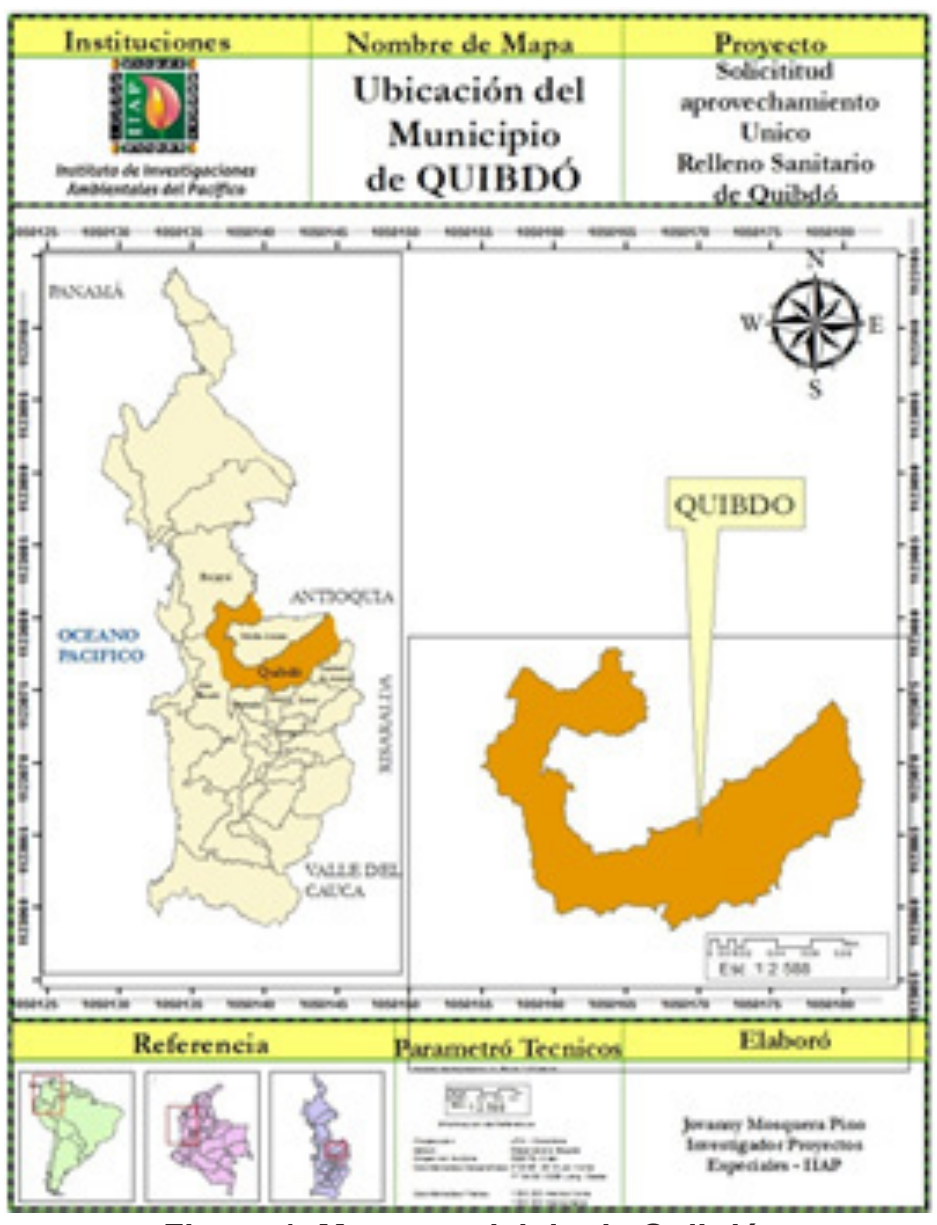

Figura 1. Mapa municipio de Quibdó.

Los valores de $11,1 \%$ determinados para la humedad de la muestra, se encuentran dentro del rango permitido y coinciden con lo dicho por el Instituto Colombiano Agropecuario (ICA), que ha determinado que para las materias primas y la producción de alimento animal, este parámetro debe estar en un rango mínimo

Tabla 1

Parámetro fisicoquímico vs. método de análisis empleado*

\begin{tabular}{ll}
\hline \multicolumn{1}{c}{ Parámetro analizado } & Método \\
\hline Humedad (perdida por secado) & GTC 1.14 Agua (humedad), determinación \\
\hline Cenizas o minerales totales & AOAC 923.03. Determinación de cenizas \\
Grasa total & GTC 6.1 Grasa bruta (extracto etéreo), determinación \\
\hline Nitrógeno total & AOAC 954.01(Coeficiente 6.25) método Microkjeldhal \\
Proteína total & AOAC 954.01(coeficiente 6.25) método Microkjeldhal \\
Carbohidratos totales & Cálculo a partir de componentes \\
Calorías totales & Cálculo a partir de componentes \\
\hline
\end{tabular}

* Laboratorio de Salud Pública, Universidad de Antioquia 
Bioetnia Volumen 10, 2013

Tabla 2

Aspectos físicos de la composición alimenticia

\begin{tabular}{lll}
\hline Parámetros físicos & \multicolumn{1}{c}{ Resultado } & Interpretación \\
\hline Humedad & $11,1 \%$ & Rango esperado \\
Color & Beige & Normal \\
Olor & Inobjetable & Normal \\
Aspecto o forma & Polvo amorfo heterogéneo & No peletizado \\
\hline
\end{tabular}

Fuente: Mosquera M. IIAP, 2014.
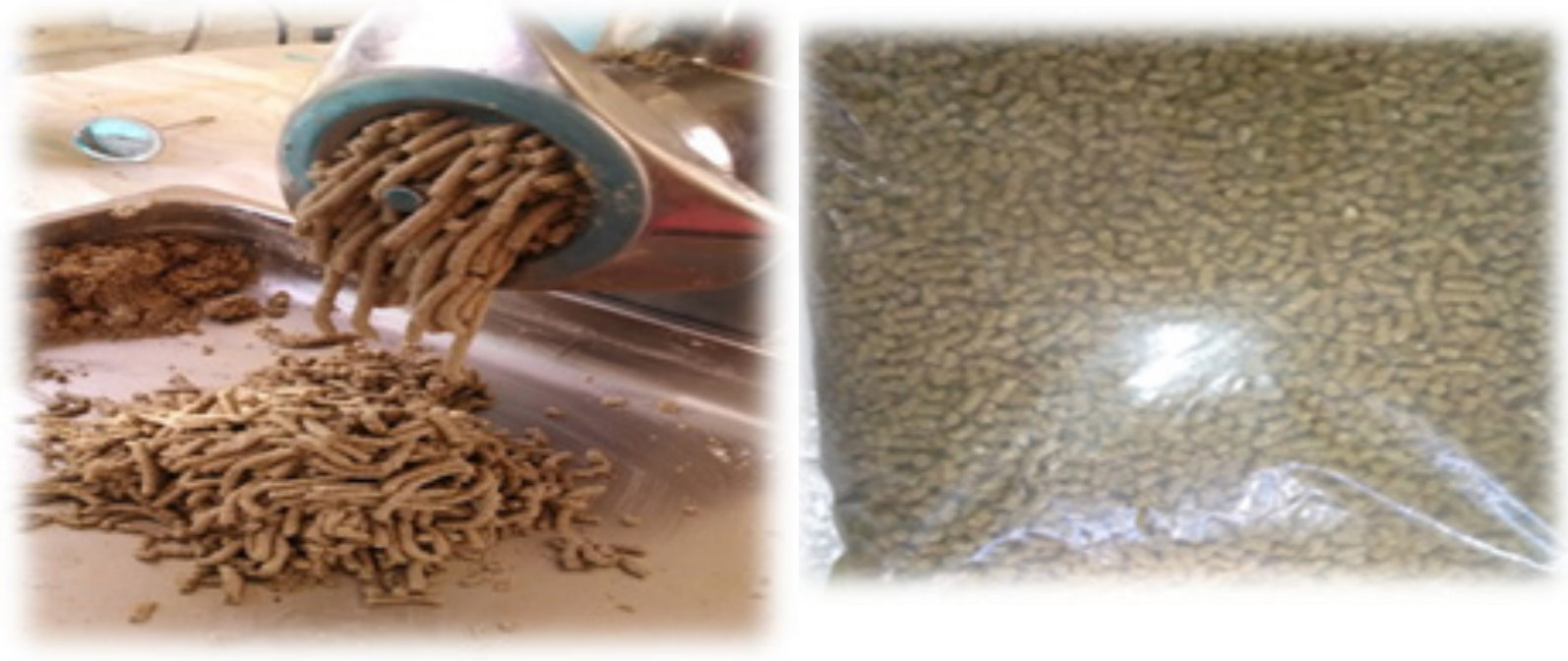

Figura 2. Preparación y empacado Pellets, composición alimenticia.

de $10 \%$ y máximo de $15 \%$.

Un alto porcentaje de humedad presente en el alimento, es indicativo de un mal almacenaje o cosecha de la materia prima, pone en riesgo su conservación, promocionando la aparición de organismos fungosos causantes de enfermedades diarreicas en el animal y aumenta los costos de producción en explotaciones comerciales avícolas.

El color beige y el olor inobjetable, determinados con la composición se asocia con los colores y olores presentes en las materias primas utilizadas, sobre todo las harinas de maíz y plátano, que tienen un efecto en la coloración de la piel y carne del animal, así como fundamentalmente en el color del huevo en el caso de gallinas ponedoras, lo cual corrobora lo dicho por Soler et al. (2011), quienes sostienen que las diferencias en el color de la carne, la consistencia y composición de la yema de huevo puede deberse a la alimentación de la gallina. Como la yema tiene un alto porcentaje de lípidos en su composición, la asimilación de pigmentos liposolubles modificará el color de la yema. Así, se encuentran yemas de colores que van desde el amarillo pálido hasta el anaranjado intenso. Algunas materias primas como el maíz o la alfalfa contienen xantofilas, que darán el color característico a la yema y la carne del ave, pero también es posible suministrar los pigmentos adecuados para obtener el color deseado. Así, la combinación de zeaxantina y de luteína con capsantina o análogos sintéticos en las dosis adecuadas modificará el color de la yema y carne, de forma que se cumplirán las expectativas del consumidor.

Con respecto al aspecto o forma del alimento, la muestra enviada al laboratorio presentó una condición amorfa y heterogénea porque para el objeto de los análisis requeridos no era necesario enviar más allá que la mezcla de ingredientes. Como producto final del estudio se elaboró una presentación comercial (Peletizado) de la composición alimenticia, con tamaño promedio de los pellet de 350 a 450 micrones, la cual fue empacada, tal como lo muestra la Figura 2.

La forma del alimento suministrado a las aves es importante por el tipo de boca característico de estas, el pico, es la única estructura que tienen las aves para procesar los alimentos, los cuales tragan entero.

Las aves de corral realizan una selección de su alimento prefiriendo harinas gruesas, sobre las finas (Nir et al. 1994). 
Variables fisicoquímicas y bromatológicas. M Mosquera et al.

Tabla 3

Análisis comparativos de los contenidos nutricionales de la composición alimenticia vs. alimento comercial

\begin{tabular}{|c|c|c|c|}
\hline \multirow[t]{2}{*}{ Fuentes / Parámetros } & \multicolumn{2}{|c|}{ Composición alimenticia } & \multirow{2}{*}{$\begin{array}{c}\text { Concentrado comercial } \\
\text { Contenido (\%) }\end{array}$} \\
\hline & Contenido (\%) & Interpretación & \\
\hline Cenizas o minerales totales & $37,62^{*}$ & Alto & 8.0 \\
\hline Grasa total & $24,82^{*}$ & Alto & 2.5 \\
\hline Nitrógeno total & 2,52 & Normal & 0.0 \\
\hline Proteína total & 15,73 & Normal & 19.0 \\
\hline Fibra & No determinado & No determinado & 5.0 \\
\hline Carbohidratos totales & 10,70 & Normal & 0.0 \\
\hline Calorías totales (Kcal/100 g) & 329,04 & Alto & 0.0 \\
\hline
\end{tabular}

Fuente: Mosquera M, IIAP, 2014

Esta característica de granulometría gruesa del alimento favorece la funcionalidad de la molleja, permitiendo un tiempo más prolongado de exposición del alimento a las enzimas digestivas y una mejor condición de acidez en el tracto digestivo. Estas condiciones permiten que los nutrientes contenidos en las dietas tengan un eficiente aprovechamiento, resultando en una mayor proporción de nutrientes disponibles a nivel intestinal (Brito 2009).

Otros autores han demostrado que con alimentos que contengan un tamaño de partícula más gruesa se tiene una mejor digestión para satisfacer los requerimientos de mantenimiento y sobre todo una alta disponibilidad de estos nutrientes para producción (Parsons et al. 2006).

Magro y Penz (1998), demostraron que en pollos de engorde en la fase de crecimiento, al incrementar el tamaño de partícula de 300 a 600 micrones en las dietas, se mejoró el resultado zootécnico. El tamaño de los órganos internos y la cantidad de carne de pechuga también mejoraron.

Por lo general se obtiene mejor crecimiento y eficiencia alimenticia cuando el alimento se dispone en migajas o minipelets (iniciación), mientras que las raciones de crecimiento y finalización se elaboran en forma de pellets. Es innegable que cuando las migajas o pellets son de mala calidad, los animales no lo consumen y decae el rendimiento, por lo que en las fincas y granjas se debe prestar suma atención al manejo del alimento para evitar que se desbarate.

Parámetros bromatológicos. La Tabla 3 muestra los resultados sobre el contenido nutricional de la composición alimenticia propuesta por el IIAP y las diferencias con respecto al alimento concentrado comercial. De los resultados anteriores se destacan la marcada diferencia entre los contenidos de minerales, grasas, carbohidratos y calorías totales de la composición alimenticia con respecto al concentrado comercial.
La Tabla 3 muestra claramente una alta concentración de minerales totales en la composición alimenticia, con $29,6 \%$ más que el alimento concentrado; esta diferencia se debe fundamentalmente al contenido de minerales en las materias primas utilizadas en la dieta propuesta por el IIAP, como son harina de hueso (10\%) y carbonato de calcio (6\%), adicionalmente la harina del maíz seco también contiene porcentajes elevados de minerales como el potasio (287 mg), magnesio (127 mg), hierro (2,7 mg), fósforo (210 $\mathrm{mg})$, manganeso $(0,48 \mathrm{mg})$; zinc $(2,21 \mathrm{mg})$ y selenio $(15,5$ $\mathrm{mcg}$ ), contiene vitamina A (469 UI), vitamina B1-tiamina $(0,38 \mathrm{mg})$, vitamina $\mathrm{B} 2(0,20 \mathrm{mg})$, vitamina $\mathrm{E}(0,78 \mathrm{mg})$ y niacina $(3,62 \mathrm{mg})$.

Asimismo, el plátano contiene altas concentraciones de minerales, sobre todo fósforo $(23 \mathrm{mg})$, hierro $(0,59 \mathrm{mg})$, yodo $(2,4 \mathrm{mg})$, magnesio $(36,4 \mathrm{mg})$, manganeso $(0,29$ $\mathrm{mg})$, calcio $(7,30 \mathrm{mg})$ y potasio $(370 \mathrm{mg})$ (propiedades del plátano). Los minerales y las vitaminas son necesarios y básicos para todas las funciones metabólicas y prevención de enfermedades. El calcio y el fósforo son elementos nutricionales presentes en la dieta propuesta e indispensable para la formación de los huesos y el manganeso en la formación de los músculos del animal.

Los resultados sobre la concentración de minerales como el fósforo y el calcio, corroboran lo expuesto en la guía de manejo del pollo de engorde Arbor Acres (2009), en donde se sostiene que el calcio de la dieta influye el crecimiento, la eficiencia alimenticia, el desarrollo óseo, la salud de las piernas, el funcionamiento de los nervios y el sistema inmune, por lo cual es vital aportar el calcio en las cantidades adecuadas y en forma consistente. $\mathrm{Al}$ igual que este, el fósforo se requiere en la forma y la cantidad correctas para la estructura y el crecimiento óptimos del esqueleto. 


\section{Bioetnia Volumen 10, 2013}

De acuerdo con Gibert (2009), los minerales son nutrientes especiales para los animales y deben estar presentes en la cantidad adecuada; esto permite que por ejemplo, en pollos parrilleros se alcancen pesos de 2,2 kilos en 42 días, lo cual requiere altas demandas y consumos de minerales que deben estar compensadas y disponibles, de tal forma que ellos puedan mantener su vida, salud y estado productivo. Esto corrobora los resultados obtenidos con la composición nutricional porque se alcanzaron pesos promedios de 4,5 libras entre 45-50 días durante las diferentes pruebas pilotos realizadas.

De otro lado, las grasas totales registraron $24,8 \%$, valor muy superior al 2,5\% del alimento concentrado, esta diferencia está asociada con los altos contenidos de ácidos grasos esenciales poli y monoinsaturados $(4,7 \mathrm{~g} / 100$ g pulpa) y las calorías presentes en el maíz, así como de grasas insaturadas en proporción menor en el plátano. En consideración de botanical.online, el maíz es rico en ácidos grasos poliinsaturados, superado sólo por el aceite de nueces, este cereal contiene $24,2 \%$ de ácido oleico (monoinsaturado), 58,7\% de ácido linoleíco (omega 6) y solo $12,7 \%$ de grasas saturadas.

A su vez, el plátano tiene muy bajo contenido de grasas $(0,27 \mathrm{~g} / 100 \mathrm{~g}$ pulpa $)$ pero una alta concentración de hidratos de carbono, lo cual le da la propiedad de coadyuvar a la ganancia de peso en el animal, esto explica los bajos o nulos contenidos de grasa y colesterol acumulados en la carne y tejidos de los pollos alimentados con esta composición alimentaria, obteniendo hasta $95 \%$ de canal sin bolsas de grasa acumulada (carne magra).

Los resultados anteriores son concordantes con lo dicho por Cabel et al. (1988), quienes sostienen que las grasas de buena calidad (no saturadas) son de excepcional valor en los alimentos de las aves. Las grasas no solo son fuentes concentradas de energía, sino también mejoran el canal, reducen el polvo en el alimento, mejoran la palatabilidad y bajan las necesidades en energía para el peletizado. Al contrario, las grasas de calidad pobre (saturadas) pueden ser extremadamente deletéreas a la producción aviar.

Las calorías totales presentes en la muestra analizada sumaron 329,04 Kcalorías/100 g, lo que es explicable por el alto nivel de calorías presentes en las harinas de maíz y un más bajo contenido en la de plátano, base de la composición nutricional ya que contienen 365 y 92 Kcalorías/100 g respectivamente, ratificando lo sostenido por el Ministerio de Agricultura y el Departamento Administrativo de Estadísticas de Colombia (DANE), sobre los requerimientos necesarios por el animal que van entre 3.100 a 3.900 Kcalorías/ciclo, así como que los alimentos completos balanceados para las aves de corral buscan mantener la actividad metabólica de los animales y permitir que cumplan con su finalidad productiva, es por esto que se componen de una mezcla de materias primas que aportan diferentes componentes. Así pues, los cereales, sobre todo el maíz y sorgo, proporcionan energía, mientras que la harina de soya y menos frecuentemente harinas de subproductos de origen animal se integran por su aporte de proteínas y aminoácidos.

De acuerdo con estas entidades, el maíz es el cereal más utilizado para la elaboración de alimentos completos balanceados para aves en Colombia, por ser una buena fuente de energía disponible debido su alto contenido de almidón y grasa. También se destaca por su palatabilidad y bajo contenido de factores antinutricionales, lo que garantiza una buena aceptación y consumo de parte de los animales. Su contenido de proteína es bajo, así como su concentración de minerales.

Costos de la composición alimentaria. La realización de los cálculos tuvo en cuenta los costos para la producción de 75 pollos de 8 días de nacidos, un ciclo productivo de 45-50 días y se obtuvo a partir de multiplicar la cantidad de alimento consumido por las aves/ciclo y los costos de los ingredientes que conforman la dieta alternativa. La Tabla 4, describe los insumos y costos de cada una de las materias primas utilizadas. Posteriormente se compararon con los precios de ventas del concentrado comercial en los mercados del municipio de Quibdó. Se destaca en la preparación de la composición alimenticia el uso de especies vegetales que tradicionalmente han sido cultivadas en los huertos productivos de las comunidades locales, utilizadas comúnmente para la alimentación humana y animal en todo el Chocó Biogeográfico.

Los resultados del análisis de la Tabla 4 muestran contundentemente la diferencia de costos entre las fuentes alimentarias que se han estudiado en esta investigación, se evidencia que cuando se utiliza el concentrado comercial se tienen costos totales por valor de $\$ 234.934$ pesos, mientras que para el caso de la composición alimenticia se requieren $\$ 197.627$ pesos, lo cual genera una diferencia u ahorro de $\$ 37.037$ pesos por concepto del alimento/ciclo productivo.

Se observa también, que a pesar de que se consumen 341 libras/ciclo de la composición alimenticia frente a 329 libras del concentrado comercial, los bajos costos de las materias primas de la composición hacen menos costoso y mejoran la rentabilidad de la producción de pollos de engorde bajo las condiciones agroambientales y tecnológicas locales.

Los resultados alcanzados ratifican lo manifestado por Abiodun y Falade (2011), quienes sostienen que el principal objetivo en el procesamiento de alimentos es el aprovechamiento de una materia prima económica, de alta durabilidad y elevada cantidad de compuestos con valor alimenticio, con el fin de reducir los costos potenciales y evitar su deterioro.

Se ratifica asimismo, lo dicho por Yomeni et al. (2004), 
Variables fisicoquímicas y bromatológicas. M Mosquera et al.

Tabla 4

Análisis de costos entre el concentrado comercial vs. composición alimenticia (Ciclo productivo entre 45 y 50 días)

\begin{tabular}{lccc}
\hline T1 (concentrado comercial) & Cantidad libras & Valor libra (\$) & Valor total (\$) \\
\hline Total costo del tratamiento & 329,5 & 713 & 234.934 \\
& & & \\
T2 (composición alimenticia) & Cantidad libras & Valor libra (\$) & Valor total (\$) \\
Harina de maíz 25\% & 85,25 & 600 & 51.150 \\
\hline Harina de plátano 33\% & 112,53 & 175 & 19.693 \\
Torta de soya 25\% & 85,25 & 1000 & 85.250 \\
Harina de hueso 10\% & 34,1 & 1000 & 34.100 \\
Carbonato de calcio 6\% & 20,46 & 280 & 5.729 \\
\hline Sal 1\% & 3,41 & 500 & 1.705 \\
\hline Total costo del tratamiento & 341 & & 197.627 \\
\hline
\end{tabular}

Fuente: Mosquera BM. IIAP, 2015

en relación a que se debe establecer como prioridad la búsqueda de nuevas alternativas alimenticias para incrementar la rentabilidad y su conservación.

De acuerdo con lo publicado por Broiller-Ross (2009), el alimento es uno de los principales componentes del costo total de producir pollos de engorde. Las raciones se deben formular para aportar el balance correcto de energía, proteína y aminoácidos, minerales, vitaminas y ácidos grasos esenciales, para permitir el crecimiento y rendimiento óptimos.

\section{Conclusiones}

Los resultados de los análisis bromatológicos a la composición alimenticia permitieron determinar sus características físicas y químicas, así como establecer diferencias significativas en cuanto a las concentraciones de nutrientes respecto al concentrado comercial.

Las variables físicas de la composición alimenticia, como lo es humedad (11,1\%), color (beige) y olor inobjetable, no difieren significativamente de las del concentrado comercial y se encuentran dentro de los parámetros requeridos por el ICA para la preparación y comercialización de alimento para pollos de engorde, garantizan la conservación e inocuidad del alimento y la ingesta por parte de los animales, generando con ello, ventajas comparativas porque se reducen los costos de producción al no requerir insumos adicionales para su elaboración.

La presentación final o forma comercial de la composición alimenticia, está dentro de los parámetros recomendados por el Instituto Colombiano Agropecuario como lo son Pellets, con tamaños entre 350 y 450 micrones.
La composición alimenticia mostró una alta concentración de minerales totales $(37,6 \%)$, lo que representa $29,6 \%$ más que en el alimento concentrado comercial; esta diferencia se debe fundamentalmente al contenido de minerales presentes en las materias primas utilizadas en la dieta propuesta por el IIAP, como son harina de hueso (10\%) y carbonato de calcio (6\%); adicionalmente la harina del maíz seco también contiene porcentajes elevados de minerales como el potasio $(287 \mathrm{mg})$, magnesio $(127 \mathrm{mg})$, hierro (2,7 mg), fósforo (210 mg), manganeso (0,48 mg); zinc $(2,21 \mathrm{mg})$ y selenio $(15,5 \mathrm{mcg})$. Asimismo, el plátano aporta también altas concentraciones de minerales, sobre todo fósforo $(23 \mathrm{mg})$, hierro $(0,59 \mathrm{mg})$, yodo $(2,40 \mathrm{mg})$, magnesio $(36,4 \mathrm{mg})$, manganeso $(0,29 \mathrm{mg})$, calcio $(7,30$ $\mathrm{mg})$ y potasio $(370 \mathrm{mg})$.

El maíz garantiza el suministro de vitaminas como vitamina A (469 UI), vitamina B1-Tiamina $(0,38 \mathrm{mg})$, vitamina B2 $(0,20 \mathrm{mg})$, vitamina $\mathrm{E}(0,78 \mathrm{mg})$ y niacina $(3,62 \mathrm{mg})$, por lo que no se requiere comprar estos suplementos para la preparación de la composición alimenticia, lo que se refleja favorablemente en los costos de producción.

La composición alimenticia registró una alta concentración de las grasas totales $(24,8 \%)$, valor muy superior al $2,5 \%$ presente en el concentrado comercial; esta diferencia está relacionada con los altos contenidos de ácidos grasos esenciales poli y monoinsaturados $(4,7 \mathrm{~g} / 100 \mathrm{~g}$ pulpa) presentes en el maíz, así como de grasas insaturadas en proporción menor en el plátano. El maíz es rico en ácidos grasos poliinsaturados, contiene $24,2 \%$ de ácido oleico (monoinsaturado), 58,7\% de ácido linoleico (omega 6) y solo $12,7 \%$ de grasas saturadas, lo que se denota claramente 


\section{Bioetnia Volumen 10, 2013}

en la relación canal y muy baja grasa presente en la carne magra de los pollos alimentados con esta dieta.

Las calorías totales presentes en la muestra analizada sumaron 329,04 Kcalorías/100 g, lo que es explicable por el alto nivel de calorías presentes en las harinas de maíz y un más bajo contenido en la de plátano, base de la composición nutricional porque contienen 365 y 92 kgcalorías/100 g respectivamente, ratificando lo sostenido por el Ministerio de Agricultura y el Departamento Administrativo de Eestadísticas de Colombia (DANE), sobre los requerimientos necesarios por el animal que van entre 3.100 a 3.900 Kcalorías/ciclo.

Se determinó una alta diferencia entre los costos de las fuentes alimentarias utilizadas en este estudio, porque cuando se utilizó el concentrado comercial se generaron costos totales por valor de $\$ 234.934$ pesos, mientras que para el caso de la composición alimenticia se requirieron $\$ 197.627$ pesos, lo que establece una marcada diferencia de $\$ 37.037$ pesos por concepto del alimento por ciclo productivo y que contundentemente reducen favorablemente los costos de producción en explotaciones avícolas bajo estas condiciones de trabajo.

A pesar de que se consumen 341 libras/ciclo de la composición alimenticia frente a 329 libras del concentrado comercial, los bajos costos de las materias primas de la composición hacen menos costoso y mejoran la rentabilidad de la producción de pollos de engorde bajo las condiciones agroambientales y tecnológicas locales.

\section{Literatura citada}

Abiodun-Solanke A, Falade K. 2011. A review of the uses and methods of processing banana and plantain (Musa spp.) into storable food products. J Agric Res Dev. 9: 85-96. Disponible en: http://www.revistas.unal.edu. co/index.php/acta_agronomica/rt/printerFriendly/29006/44225

Alcaldía Municipal de Quibdó. Plan de Desarrollo Municipal, 2011.

AOAC INTERNATIONAL. Official Methods of Analysis. 18th ed., 2005. Current Through Revision 3, 2010 Dr. William Horwitz.

ArborAcres. 2009. Suplemento sobre nutrición del pollo de engorde. Disponible en: http://es.aviagen.com/assets/Tech_Center/BB_Foreign_Langua-
ge_Docs/Spanish_TechDocs/AA-Plus-2009-Suplemento-Nutricin-Pollo-Engorde.pdf

BerrioAM, Cardona MG. 2001. Evaluación productiva de una dieta alternativa como reemplazo parcial de concentrado comercial en aves de postura. Unidad Municipal de Asistencia Técnica Agropecuaria (Umata), San Rafael: Facultad de Ciencias Agrarias, Universidad de Antioquia.

Botanical online. El maíz como alimento. Propiedades alimentarias del maíz. Disponible en: http://www.botanical-online.com/maizpropiedades.htm\#

Brito V. 2009. Importancia de la granulometría de los alimentos en el comportamiento productivo del pollo de engorde. Disponible en: http://www. engormix.com/MA-avicultura/nutricion/articulos/

Broiller Ross. 2009. Suplemento de nutrición del pollo de engorde. pp 21. Disponible en: http://en.aviagen.com/assets/Tech_Center/BB_Foreign_Language_Docs/Spanish_TechDocs/Ross-Suplemento-Nutricin-Pollo-Engorde-2009.pdf

Cabel M, Waldroup PW, Shermer W, Calabotta D. 1988. Effectes of ethoxyquin feed preservative and peroxide level on broiler performance. Poultry Sci. 67: 1725-30.

Departamento Administrativo de Estadísticas de Colombia (DANE). 2013. Requerimientos necesarios para la preparación de alimento animal. Boletín mensual $N^{\circ}$ 7. Bogotá: Ministerio de Agricultura y DANE.

Friedman A, Weil B. 2010. Producción avícola, negocio en crecimiento. Informe para USAID. Disponible en: http://goo.gl/vmDgvm

Gibert PM. 2009. Minerales en la nutrición de las aves. Informe de resultados del análisis físico químicos. Medellín: Universidad de Antioquia, Laboratorio de Salud Pública, Área de Análisis Fisicoquímico.

Icontec Internacional. Norma Técnica NTC-ISO/IEC Colombiana 17050-2 de 2005. Evaluación de la conformidad. Declaración de conformidad del proveedor. Parte 2: Documentación de apoyo. Bogotá: Icontec.

Instituto Colombiano Agropecuario(ICA). Proceso de producción de alimentos para animales. Bogotá: ICA.

Los alimentos. Minerales del plátano. Disponible en: http://alimentos.org. es/minerales-platano

Magro N, Penz AMJr. 1962. Efeito da granulometria da ração no desempenho e nas características teciduais de frangos de corte alimentados dos 21 aos 42 dias de idade. Poult Sci. 41: 438-44.

Mosquera BM. et al. 2008. Informe final Plan Operativo. Quibdó: Instituto de Investigaciones Ambientales del Pacífico (IIAP).

Nir I, Hillel R, Shefet G, Nitsan Z. 1994. Effect of grain particle size on performance. 2. Grain texture interactions. Poult Sci. 73: 781-91.

Parsons AS, Buchanan NP, Blemings KP, Wilson ME, Moritz JS. 2006. Effect of corn particle size and pellet textureon broiler performance in the growing. J Appl Poult Res. 15: 245-55.

Soler MD, Garcés C, Barragán JI. 2011. La alimentación de la ponedora y la calidad del huevo. Valencia: Departamento de ProducciónAnimal, Sanidad Animal, Salud Pública Veterinaria y Ciencia y Tecnología de los Alimentos, Facultad de Veterinaria de la Universidad CEU Cardenal Herrera.

Yomeni M, Njoukam J, Tchango Tchango J. 2004. Influence of the stage of ripeness of plantains and some cooking bananas on the sensory and physicochemical characteristics of processed products. J Sci Food Agric. 84: 1069-77. 